\title{
Psicología descriptiva y 'mereología' en Carl Stumpf
}

Resumen: El artículo analiza la teoría de todos y partes (mereología) de Carl Stumpf. Por un lado, se presentan los elementos básicos de su psicología descriptiva de la experiencia. Por otro lado, se analiza el desarrollo de la teoría de todos y partes sobre la base de dicha psicología descriptiva. El trabajo pretende probar dos tesis complementarias: primero, si bien la teoría mereológica de Stumpf es aún incipiente y no del todo explícita, constituye un auténtico punto de partida. Segundo, dicha mereología surge a partir de una atenta descripción de la experiencia dada y no es algo impuesto a ésta.

Palabras clave: filosofía de la experiencia, estructuras, todos, partes independientes, partes dependientes.

\begin{abstract}
The article focuses on Carl Stumpf's theory of parts and wholes (mereo$\operatorname{logy}$ ). On the one hand, I present the basic elements of his descriptive psychology of experience. On the other hand, I analyze the development of his theory of parts and wholes, which is based upon his descriptive psychology. The article aims to prove two different, yet complementary theses: firstly, despite the fact that Stumpf's mereological theory is still incipient and not quite explicit, it represents an authentic point of departure; secondly, this mereology arises out of an attentive description of given experience and not as an imposition to it.
\end{abstract}

Keywords: philosophy of experience, structures, wholes, independent parts, dependent parts.

"Doctor en Filosofía, Universidad de Colonia, Alemania; Investigador del CONICET; Profesor en la Facultad de Ciencias Jurídicas y Sociales, Universidad Nacional del Litoral; Profesor Invitado en la Facultad de Filosofía, Universidad Católica de Santa Fe. Entre otras cosas, ha publicado Absoluter Fluss - Urprozess - Urzeitigung. Die untersten Stufen der Konstitution in Edmund Husserls Phänomenologie der Zeit, Orbis Phaenomenologicus, Königshausen \& Neumann, Würzburg, 2011. Dirección electrónica: luisniel@yahoo.com 


\section{Introducción}

Antes de comenzar, quiero confesar cierto anacronismo explícito en el título del presente trabajo. El término 'mereología', es decir, la disciplina (formal) que estudia los todos y las partes, así como sus múltiples relaciones, no fue utilizado por Carl Stumpf. Ni siquiera Husserl, normalmente considerado el 'padre' de dicha disciplina, utiliza dicho concepto. ${ }^{1}$ De hecho, como veremos, en los primeros escritos de Stumpf no sólo no aparece el término 'mereología', sino que tampoco encontramos una teoría explícita de los todos y las partes, sino sólo bosquejos, insinuaciones e ideas que, a pesar de su carácter incipiente, constituyen un auténtico punto de partida que sin lugar a dudas sirvió de soporte para que Husserl formulara unos años después su teoría de todos y partes, y que luego pasará a llamarse 'mereología'. De ahí el uso de comillas en el título del trabajo. En función de lo dicho, nuestra intención es mostrar cómo, en qué términos y en qué contexto, Stumpf formuló lo que desde la perspectiva actual, sin negar dicho anacronismo, podríamos denominar 'proto-mereología'.

Carl Stumpf (1848-1936) es uno de los tantos filósofos del mundo germano parlante del siglo XIX que la tradición filosófica (y psicológica) olvidó casi por completo, pese a la enorme influencia que ejerció su pensamiento. Fue el 'segundo' maestro de Husserl (después de Brentano) y se podría quizás considerarlo como uno de los padres de la 'proto-fenomenología'. Además, sus estudios en el campo de la psicología (descriptiva y experimental) influyeron profundamente en la obra de tres de sus discípulos Wertheimer, Köhler y Koffka, los padres de la Gestaltpsychologie. Su filosofía, a veces difícil de diferenciar de la psicología, consiste en un estudio minucioso de la experiencia humana, de su funcionamiento, y del modo de constitución del mundo a partir de nuestras sensaciones y aprehensiones más básicas.

Stumpf tuvo una relación muy estrecha con Franz Brentano: fue el primer discípulo de Brentano, cuando éste aún era docente en Würzburg, antes de partir hacia Viena. Su segundo maestro fue Lotze, quien dirigió sus tesis de doctorado y de habilitación en Göttingen. Su tesis doctoral, la

\footnotetext{
${ }^{1}$ En el marco de una ontología formal, Husserl presenta su teoría 'mereológica' en la tercera investigación lógica, titulada "La doctrina de los todos y las partes" (cf. Husserl 1901/1984, pp. 227-300). Se considera en general a Stanisław Leśniewski el primero en utilizar la denominación 'mereología'.
} 
Verbältnis des Platonischen Gottes zur Idee des Guten (La relación del Dios platónico con la idea del Bien), publicada en 1869, quedó opacada tanto por su tesis de habilitación Über den psychologischen Ursprung der Raumvorstellung (Sobre el origen psicológico de la representación del espacio) de 1873 [en adelante RV], así como por su gran obra, la Tonpsychologie (Psicología de los tonos), publicada en dos volúmenes, el primero en 1883 y el segundo en 1890 [en adelante TP]. Sendas obras constituyen valiosísimos aportes a la psicología descriptiva: la primera en relación al estudio del modo de percibir (y constituir) el espacio, mientras que la segunda con respecto al modo de percepción de los sonidos e indirectamente del tiempo.

No es difícil rastrear en dichos escritos ciertos temas que tendrán una profunda influencia de la obra de Husserl -de hecho, las Investigaciones lógicas están dedicadas a Stumpf, maestro de Husserl en Halle-. Las similitudes pueden evidenciarse básicamente en el profundo respeto (fenomenológico) por la descripción de la experiencia y del modo en que se dan las diversas formas de experimentar, sea ya a partir de la sensación, de la fantasía, del juicio, etc. ${ }^{2}$ En relación al tema que nos interesa, es importante señalar la influencia de Stumpf en la teoría husserliana de los todos y las partes -el mismo Husserl reconoce a comienzos de la tercera investigación lógica que fue Stumpf una de sus principales referencias al respecto- ${ }^{3}$ En cualquier caso, no es tema del presente artículo rastrear cada uno de los puntos en los que Stumpf influyó en la obra de Husserl, sino analizar los rudimentos de la teoría de todos y partes, tal y como se desarrolla en la obra de Stumpf, en particular en sus primeros escritos, es decir, anteriores a la publicación de las Investigaciones lógicas de Husserl en 1900/01. ${ }^{4}$

\footnotetext{
${ }^{2}$ Así, por ejemplo, los análisis en torno la psicología de los tonos dejaron claras huellas en la teoría husserliana de la percepción de los objetos de tiempo, en particular, en el prototípico análisis de la percepción de una melodía (recurrente ejemplo de Husserl en sus estudios sobre la conciencia de tiempo). Lo mismo puede decirse de temas relacionados con la percepción del espacio, con la fantasía, la teoría de la rememoración, etc.

${ }^{3}$ Cf. Husserl 1901/1984, pp. 227, 233 y ss.

${ }^{4}$ Tomamos como punto de referencia la publicación de la tercera investigación de Husserl en 1901, puesto que allí Husserl formula explícitamente una teoría de todos y partes, sentando las bases de la futura mereología en tanto disciplina.
} 
El trabajo se estructura a partir de dos ejes temáticos: por un lado, presentaremos los elementos básicos de su teoría de psicología descriptiva (objeto de estudio, cuestiones metodológicas, cuestiones epistemológicas en torno a la relación con otras ciencias, cuestiones conceptuales, etc.); por otro lado, analizaremos, a partir de los elementos del punto anterior, el modo en el que Stumpf desarrolla una incipiente teoría de todos y partes. En base a estos ejes, nuestra meta será probar las siguientes tesis, que son complementarias.

Primero, Stumpf no tiene una teoría explícita y desarrollada sobre todos y partes. Sólo nos encontramos con una serie de reflexiones 'mereológicas' básicas que se hallan siempre insertas en el marco de descripciones psicológicas sobre diversos temas (el espacio, la percepción, etc.) y que constituyen el núcleo temático de sus primeras investigaciones.

Segundo, el hecho de que los conceptos 'mereológicos' no sean planteados de forma 'abstracta' en el marco de una teoría formal sobre todos y partes, responde a una convicción propia de la psicología fenomenológica de Stumpf: las relaciones de todos y partes son modos en los que se presenta la realidad. Este punto puede, a su vez, subdividirse en dos cuestiones íntimamente relacionadas: una es de índole metodológica y la otra de índole psicológico-fenomenológica. En primer lugar, Stumpf no toma como punto de partida (en sentido de anterioridad metodológica) una teoría formal para luego aplicarla a la realidad empírica; todo lo contrario, es sólo a partir de la descripción misma del modo en que se nos presentan fenómenos (tales como por ejemplo el espacio o el tiempo) que se nos muestra cuáles son las estructuras y relaciones de la realidad. En segundo lugar, Stumpf mantiene un firme compromiso con 'la cosa misma' que se refleja en su convicción de que las estructuras mereológicas son inherentes al seno mismo de la experiencia, i.e. es la experiencia la que legitima el valor de aquellas estructuras. ${ }^{5}$

Es esta cuestión de índole psicológico-fenomenológica la que da sustento a la cuestión metodológica planteada arriba.

\footnotetext{
${ }^{5}$ Barry Smith señala correctamente que para Stumpf la ontología es una parte de la psicología (cf. Smith 1994, p. 87).
} 


\section{La psicología descriptiva y su relación con la experiencia propia y con otras ciencias}

La psicología descriptiva de Stumpf es una perspectiva cuyo foco de descripción parte de aquello que puedo describir en primera persona a partir de mi propia experiencia. Tanto en sus estudios sobre la representación del espacio como en aquellos sobre los tonos, el recorrido descriptivo de Stumpf refleja una detenida y concienzuda mirada sobre los hechos más concretos de nuestra vida cotidiana; de este modo, su mirada se dirige por lo general a ejemplos muy concretos: ${ }^{6}$ cómo escuchamos una melodía, qué matices tiene, qué sucede si tapamos un oído o el otro, si nos concentramos y prestamos atención, si dejamos que fluyan las sensaciones sin una atención dirigida, etc.

Para Stumpf no hay duda de que lo más importante para una investigación científica es tener presente los "fenómenos de la conciencia ordinaria" (Phänomene des gewöhnlichen Bewnsstseins), que es aquello que sirve de fuente e inspiración para toda investigación científica (cf. Stumpf 1873, p. 106). No obstante, dicha valoración de la experiencia más básica y cotidiana, y su sesgo fenomenológico, no implica para Stumpf renunciar a los aportes de otras ciencias; cada una de éstas puede contribuir desde un enfoque que le es peculiar. En TP, Stumpf subraya la considerable diferencia de perspectivas sobre un mismo tema que pueden tener un físico, un fisiólogo y un psicólogo: así, por ejemplo, si consideramos los tonos como objeto de estudio, podemos observar que el interés de los dos primeros recae en los aspectos físicos de los mismos (exteriores, cuantificables, susceptibles de mediciones experimentales, sea ya con respecto a la acústica, a los sonidos, o bien a los procesos cerebrales con los que los captamos, etc.); al psicólogo, por otra parte, le interesa el modo en el que captamos los tonos en la experiencia propia a través de representaciones y juicios (cf. Stumpf 1883, pp. vi-vii).

En cualquier caso, para Stumpf la psicología descriptiva no debe ser indiferente a los resultados de otras ciencias: todo lo contrario, las investigaciones empíricas (exteriores), como por ejemplo los estudios llevados a cabo en los laboratorios psicológicos (muy comunes en el siglo XIX), tienen una gran relevancia para un estudio integral de un mismo fenómeno.

\footnotetext{
6 "Las relaciones [que en general estructuran nuestro mundo] no se pueden definir en un sentido estricto, sino sólo explicar a partir de ejemplos” (Stumpf 1883, p. 98).
} 
Así, en RV, Stumpf distingue dos métodos para las investigaciones psicológicas: por un lado, la observación puramente interna (rein innerliche Beobachtung), por otro, los experimentos exteriores. Ahora, cabe aclarar que si bien Stumpf reconoce la necesidad de una combinación y articulación de ambos métodos, no obstante, el segundo (el experimental) es más bien auxiliar del primero (el descriptivo) (cf. Stumpf 1873, p. 5). La clave de un auténtico sistema científico-filosófico consiste en saber articular esta psicología descriptiva con los aportes de las diferentes ciencias empíricas.

En una conferencia dictada en 1907 en la Friedrich-Wilhelms-Universität de Berlín, titulada Die Wiedergeburt der Pbilosophie (El renacimiento de la filosofía), Stumpf sostiene un profundo rechazo tanto de los sistemas de filosofía apriorísticos (a los que identifica fundamentalmente con el idealismo alemán), como de los materialismos extremos. En consonancia con dicho rechazo, su posición filosófica propone integrar la psicología descriptiva (en primera persona) con los elementos aportados por las ciencias. Contra el apriorismo idealista, Stumpf reafirma la centralidad que ocupa la propia experiencia en tanto fuente de conocimiento; contra el materialismo y el naturalismo 'reificante', propios de una mirada (experimental) en 'tercera persona', enfatiza la inevitable centralidad de mi experiencia subjetiva.

Pero es menester reiterar que no se trata de un subjetivismo que rechaza las ciencias naturales; su apuesta es no absolutizarlas (como hace cierto naturalismo), sino incluirlas en un marco más amplio, que parta de lo que me es más cercano: i.e. mi propia experiencia (cf. "Die Wiedergeburt der Philosophie" (1907), en Stumpf 1910, p. 164). En un claro distanciamiento de las 'violaciones de la realidad' (Vergewaltigungen der Wirklichkeit) que surgen del idealismo (y del misticismo generado por éste), Stumpf propone concretamente una "filosofía de la experiencia" (Erfahrungsphilosophie) (Stumpf 1910, pp. 169, 178); en este punto, coincide con la conocida metáfora de Husserl que sostiene que una auténtica filosofía se obtiene "de abajo hacia arriba" (von unten nach oben) (Stumpf 1910, p. 184). En tal sentido, Stumpf ve en la figura de su maestro, Brentano, al padre del "renacimiento de la filosofía” (Wiedergeburt der Philosophie) (Stumpf 1919, p. 88). 


\section{Cuestiones conceptuales en torno al método psicológico}

\subsection{Diferentes tipos de juicio (pasivos y activos) y sus componentes (materia y contenido)}

Para entender el método psicológico de Stumpf es menester aclarar algunas cuestiones conceptuales básicas. Uno de los conceptos centrales es el de 'juicio', que Stumpf analiza en diferentes contextos.

En TP nos encontramos con la distinción de los distintos tipos de juicios en función del grado de actividad de la conciencia: así tenemos 'juicios no reflexivos' y 'juicios reflexivos'. En función de esta distinción, se puede observar que para Stumpf el concepto de juicio (Urteil) o de (la actividad de) juzgar (Beurteilung) no remite necesariamente a una actividad judicativa explícita y formulada en un lenguaje?: es decir, no todo juicio implica una reflexión activa o una exteriorización lingüística. Así, con anterioridad a toda enunciación judicativa, hay formas más elementales de juicio que consisten simplemente en 'darse cuenta (de algo)' (Bemerken) o 'aprehender (algo)' (Auffassen); la aprehensión (Auffassung) es así una forma elemental de juzgar (algo) -término que, según Stumpf, es preferible al de 'Apperception'-.

Lo más importante aquí es la diferencia entre, por un lado, los 'juicios no reflexivos' (reflexionslose Urteile), i.e. las aprehensiones (así como los juicios de costumbre, es decir, aquellos que se basan en aprehensiones pasadas y habitualizadas), y, por otro lado, los 'juicios que surgen de la reflexión' (aus Überlegung entstehenden Urteile), que sí implican una instancia judicativa activa (cf. Stumpf 1883, pp. 5-6). Toda formulación de un juicio explícito y reflexivo (sobre algo) presupone una aprehensión pasiva y anterior de aquello que afirmamos, dudamos, negamos, etc. El enfoque utilizado por Stumpf es así genético: a partir de sensaciones (Empfindungen) $)^{8}$ o bien de la combinación y relación de sensaciones (Stumpf habla de una 'red de relaciones') se generan juicios no-reflexivos de un modo no voluntario, y sobre la base de estos juicios pre-reflexivos se fundan a su vez los juicios reflexivos (cf. Stumpf 1883, pp. 6-7).

\footnotetext{
${ }^{7}$ Es muy importante tener en cuenta este punto, considerando que a lo largo de TP el tema central son los 'juicios de tonos' (Tonurteile).

${ }^{8}$ Las condiciones empíricas de dichos juicios se fundan a su vez en las sensaciones actuales (angenblickliche Empfindungen) o en los estados actuales de la mente (augenblickliche Seelenzustande) (cf. Stumpf 1883, p. 5).
} 
En unos resúmenes de sus lecciones sobre lógica dictadas en 1886/87 (cf. Stumpf 1886/87b) nos encontramos con la distinción de los componentes del juicio, que son la 'materia' y el 'contenido'.

(a) La 'materia' (Materie) del juicio son los 'objetos' juzgados con independencia del carácter aseverativo del juicio (i.e. de su afirmación o negación); éstos pueden ser tanto lo dado en una representación (Vorstellung) (así, por ejemplo, cuando decimos 'Dios existe', 'Dios' es la materia del juicio), o bien lo dado en una relación (por ejemplo, 'la igualdad de 4 y $2+2$ '); aún en este último caso, la referencia es a la relación en tanto objeto dado, es decir, sin afirmación o negación.

(b) El 'contenido' (Inhalt) del juicio ${ }^{10}$ es aquello que Stumpf denomina 'estado-de-cosas' (Sachverhalt) ${ }^{11}$, i.e. aquello que se afirma o se niega en un juicio; retomando los ejemplos anteriores, cuando decimos 'Dios existe', el contenido del juicio o el estado-de-cosas al que se refiere el juicio es precisamente 'el existir de Dios' (o bien, en el segundo caso, es el 'afirmar que 2 +2 es 4'. Así, si decimos 'Dios existe' o bien 'Dios no existe', tenemos dos juicios que tienen la misma materia (i.e. Dios), pero distintos contenidos (i.e. el existir / el no-existir de Dios) (cf. Stumpf 1886/87b, pp. 312-313).

\subsection{Representaciones y relaciones}

Como mencionamos en el párrafo anterior, la 'materia' del juicio (i.e. los 'objetos' que componen el juicio) puede estar compuesta por representaciones o relaciones.

\footnotetext{
${ }^{9}$ Dichos resúmenes fueron dados por Stumpf a sus estudiantes en la Universidad de Halle, para que los mismos pudieran seguir mejor las lecciones. Husserl tenía una copia de éstos que se conserva en los Archivos de Lovaina (cf. Stumpf 1886/67a, p. 283).

${ }^{10}$ Es menester subrayar aquí la diferencia entre el 'contenido del juicio', que es aquello a que refiere el juicio, y el 'contenido de las representaciones', que son todos aquellos contenidos mentales que vienen dados por las sensaciones, la fantasía, la memoria, etc.

${ }^{11}$ Stumpf fue el primero en utilizar el concepto técnico de 'Sachverhalt', que tuvo un rol esencial en la filosofía del siglo XX. Si bien hay algunos antecedentes históricos (por ejemplo, Brentano hablaba de 'contenido de juicios' y Lotze también utilizó una categoría similar), se puede decir con certeza que fue Stumpf quien introdujo el término con un sentido técnico preciso.
} 
(i) Las representaciones (Vorstellungen) ${ }^{12}$ son los 'contenidos absolutos' (absolute Inhalte) $)^{13}$ de la conciencia, en particular los contenidos de sensaciones y en un segundo sentido podemos hablar de contenidos de la fantasía, de la memoria, etc. Son diversos los modos cognitivos de 'reaccionar' a dichos contenidos: así, por ejemplo, una 'percepción' (Wahrnehmung) es afirmar o darse cuenta de un contenido; el contenido de percepción puede ser, no obstante, el resultado de un engaño (Täuschung) -i.e. una percepción puede ser de hecho engañosa y luego corregirse por otra ${ }^{14}-$. En el caso de percepciones ciertas hablamos de 'conocer' (Erkennen).

(ii) Pero lo que nos interesa en particular son las relaciones: éstas también son materia de juicios. Así, podemos de hecho percibir ciertas relaciones (Stumpf utiliza casi indistintamente los términos germánicos 'Verbältnisse' y 'Beziehungen'), como por ejemplo: pluralidad, incremento, similitud, fusión, etc. ${ }^{15}$ Stumpf llama 'análisis' (Analyse) al darse cuenta (afirmar, percibir) de una pluralidad, y 'relacionar' (Beziehen) o 'comparar' (Vergleichen) al darse cuenta de una similitud, de una fusión, etc. (cf. Stumpf 1883, p. 96 y ss.). Volveremos luego sobre este tema.

La conclusión a la que debemos llegar aquí es que la experiencia y, correlativamente, los juicios que hacemos sobre ella (por supuesto, aún en

\footnotetext{
${ }^{12}$ Es importante aquí señalar ciertos inconvenientes en la traducción del término alemán 'Vorstellung'. Históricamente, el término fue interpretado (tanto en la obra de racionalistas como de empiristas modernos) en tanto 'idea' que hace las veces de mediación entre el sujeto y el mundo (tema duramente cuestionado por Heidegger). No obstante, fue Brentano quien dio otro sentido a dicho concepto: 'Vorstellung' es así una 'presentación' o ‘dación' directa de la cosa en el marco de mi experiencia de mundo. Stumpf también se ubica en el marco de dicha concepción brentaniana. Por razones que obedecen a una larga tradición en la traducción, hemos optado por mantener el término 'representación', haciendo esta salvedad en cuanto a su significación.

${ }^{13}$ En base a lo dicho arriba (cf. supra, nota 10), debe quedar claro que aquí nos referimos a los 'contenidos de las representaciones' y no a los 'contenidos de los juicios'.

${ }^{14}$ Es conocido el ejemplo de Husserl de la muñeca: a la distancia creemos (mediante una percepción) que es una persona, pero, al acercarnos a ella, nos damos cuenta de que es una muñeca.

${ }^{15}$ Podemos observar aquí un cierto bosquejo, avant la lettre, del concepto husserliano de 'intuición categorial'.
} 
el sentido más básico, en tanto juicios no reflexivos) no sólo nos presentan contenidos individuales, como por ejemplo un dato de sensación, sino además relaciones de diversa indole. Ésta es quizás una de las principales enseñanzas de TP: el estudio de la psicología de los tonos, i.e. de los juicios (en sus diversos niveles) que tenemos sobre los tonos, sean éstos sucesivos (por ejemplo, en una escala tonal) o simultáneos (por ejemplo, en un acorde), nos muestra las relaciones que configuran la realidad. En otras palabras: analizar el modo psicológico en el que captamos tonos nos lleva a darnos cuenta de que nuestra mirada no puede reducirse a meros contenidos (datos de sensación) y objetos, sino que la realidad se compone a su vez de múltiples relaciones. Pero hay un tema importante que aún debemos precisar en torno al 'análisis' mencionado arriba: ¿en qué sentido hablamos de acontecimientos (meramente) pasivos (passive Ereignisse) y en qué sentido de actividades (Thätigkeiten) de la conciencia?

\subsection{Las relaciones entre pasividady actividad, y el concepto de análisis}

El concepto de 'análisis' atraviesa diferentes niveles que van desde acontecimientos de tipo pasivo a actos reflexivos de la conciencia. Para entender bien esto, es necesario precisar primero algunas cuestiones conceptuales. Stumpf distingue la 'sensación' (Empfindung) de la 'aprehensión’ (Auffassung). ${ }^{16}$ Como sostiene en el Prólogo del segundo tomo de TP, lo que nos es dado en nuestra experiencia diaria son las "sensaciones en aprehensiones" (Stumpf 1890, p. vi). Es decir, normalmente las sensaciones se dan conjuntamente con aprehensiones de las mismas; las sensaciones 'a secas' no son más que una suerte de 'murmullo de fondo' (si se me permite la metáfora), al que constantemente vamos dando significación a partir de aprehensiones. En base a esto, el método psicológico-descriptivo debe proceder analíticamente, descomponiendo estas vivencias en sus componentes (sensaciones y aprehensiones).

La 'sensación' (Empfindung) es todo aquello que me viene dado por los sentidos. ${ }^{17} \mathrm{En}$ TP, Stumpf hace alusión fundamentalmente a las sensaciones

\footnotetext{
16 Nuevamente, encontramos aquí una importante analogía conceptual con la fenomenología de Husserl, en particular con el conocido 'esquema contenidoaprehensión’ al que Husserl se refiere a lo largo de su obra.

${ }^{17}$ Las sensaciones son "representaciones (Vorstellungen) que surgen desde afuera por
} 
auditivas, mientras que en RV a las sensaciones visuales y táctiles. Cuando hablamos de 'contenido' de las sensaciones, no debe malinterpretarse esto y entenderlo en tanto algo 'cosificado'; todo lo contrario, un contenido de sensación es algo dado en mi experiencia subjetiva: es un 'sentir' (Empfinden) que, a al mismo tiempo, es 'algo sentido' (Empfundenes).

La 'aprehensión' (Auffassung) es la captación involuntaria (unwillkürlich) de aquello dado en una sensación. Por ejemplo, cuando escuchamos dos tonos diferentes, nos damos cuenta de la diferencia. Dicho darse cuenta implica una captación aprehensiva de la mentada diferencia, que se presenta conjuntamente con las sensaciones de dichos tonos (cf. Stumpf 1883, p. 105). Como mencionamos arriba, lo que no hay que olvidar es que la aprehensión, tal y como la entiende Stumpf, no es necesariamente algo activo, determinado voluntariamente por el sujeto. Por el contrario, la aprehensión es la captación básica de diferencias, similitudes, etc. al nivel de las sensaciones, i.e. que linda entre pasividad y un grado mínimo de actividad. Dicho en otras palabras: mientras que las sensaciones son un mero darse (algo) a los sentidos (i.e. un dato sensitivo), la aprehensión es el 'darse cuenta' elemental de la conciencia, es decir, es captar ese algo sensible (muy común en nuestra experiencia cotidiana), casi sin darnos cuenta de un modo activo (cf. Stumpf 1883, p. 107). Por ejemplo, cuando escuchamos una sinfonía de Brahms, no tenemos meras sensaciones aisladas, sino que captamos aprehensivamente los sonidos orquestales en sus unidades de sentido, sus altos y bajos, su matices, etc. Por otra parte, dicha captación no implica (necesariamente) una detenida atención dirigida explícitamente a captar, por ejemplo, el rol de cada uno de los diferentes instrumentos, las combinaciones melódicas y armónicas, los contrapuntos, etc.; esto último es más bien el resultado de una captación activa, fruto de un dirigir la atención (Zuwendung der Aufmerksamkeit) hacia aquello ya captado aprehensivamente en un sentido básico, pasivo. Este segundo momento, que podríamos llamar 'atencional', es una aprehensión de tipo reflexiva, atenta, i.e. un acto secundario, que sobreviene al acto primario de aprehensión involuntaria.

la mediación de los órganos externos” (Stumpf 1886/87a, p. 291). En este pasaje, Stumpf también denomina 'sensaciones', en un segundo sentido, a todo proceso neuronal que se genera a través de la actividad muscular. 
Con el concepto de 'análisis' sucede algo similar. Dijimos arriba (cf. supra, Cap. 2.2) que el 'análisis' es un juicio (no necesariamente reflexivo, explícito, lingüístico) a través del cual se capta y percibe una multiplicidad. Stumpf distingue dos formas de 'análisis': en sentido básico (elemental), el 'análisis' es un proceso de descomposición (Zerlegungsvorgang) que efectúa la conciencia, a partir del cual captamos los elementos que componen una pluralidad en un sentido pasivo (o en un sentido de actividad muy elemental), por ejemplo, cuando escuchamos los tonos de una melodía. En sentido estricto, hablamos de 'análisis' cuando diferenciamos activamente los elementos, es decir, cuando en virtud de la dirección de la atención distinguimos los elementos que se encuentran bajo consideración de un modo voluntario; retomando el ejemplo anterior, hablamos de análisis en sentido estricto cuando descomponemos una obra musical ${ }^{18}$ (cf. Stumpf 1883, pp. 96-97). Ahora, es fundamental subrayar que este segundo sentido (estricto) de 'análisis' depende del primero (pasivo), en tanto es el que lo hace posible dándole la base necesaria para operar con distinciones más complejas.

¿A qué conclusión llega entonces Stumpf y por qué este recorrido de los elementos básicos del funcionamiento de la conciencia (y correlativamente del método psicológico descriptivo) es relevante para nosotros? La conclusión a la que debemos llegar es la siguiente: tanto la sensación como la aprehensión de la misma no son resultado de una actividad voluntaria, sino que, por el contrario, es algo que nos viene dado por la naturaleza misma de la experiencia que recibimos; a decir de Stumpf, las sensaciones y aprehensiones (básicas): "se meten dentro de nosotros, el no-yo en el yo" (sie setzen sich selbst in uns binein, das Nichtich in das Ich) (Stumpf 1883, p. 105). Más allá de ciertas resonancias fichteanas de la oración (que Stumpf descartaría por completo), la idea es indicar que no sólo la sensación se trata de algo dado, sino que nuestro modo subjetivo de 'reaccionar' ante la misma (aprehensión, análisis) es un modo pasivo e involuntario de 'captar' ese algo que se da en la experiencia misma. En otras palabras: las sensaciones y sus respectivas aprehensiones, así como las relaciones entre éstas nos vienen dadas por la realidad misma. La psicología descriptiva nos muestra de este modo que nuestra experiencia no es el resultado de una imposición de estructuras conceptuales

${ }^{18}$ Evidentemente, en este marco también podemos diferenciar distintos niveles de análisis que van desde análisis reflexivos más informales a formas complejas de análisis científico. 
formales a la realidad de un modo exógeno. Todo lo contrario, es la realidad misma la que se nos impone a partir de nuestra experiencia y ésta es el criterio último de validación de toda estructura conceptual. Es en este contexto de comprensión que Stumpf formula su teoría (mereológica) de las relaciones que, así como las sensaciones, nos son dadas en la realidad misma.

\section{El análisis de tipo estructural-conceptual y su objeto}

\subsection{La investigación genética y la investigación estructural-conceptual}

En su juvenil trabajo de habilitación (la RV de 1873), Stumpf presenta un minucioso estudio sobre el problema del espacio. Como confiesa el mismo autor en el prólogo de la obra, su investigación se inscribe en el marco de los debates de los nuevos estudios psicológicos y fisiológicos que crecieron en popularidad a lo largo del siglo XIX (defendiendo una filosofía científica y positiva), muchos de ellos, en relación contestataria con respecto a la filosofía de Kant (y a fortiori como reacción al idealismo alemán). De hecho, es la doctrina del espacio de Kant, en tanto forma a priori subjetiva, el punto de partida de su investigación, pasando luego por la obra de otros pensadores del siglo XIX, tales como Herbart, Bain, Weber y Lotze. En cualquier caso, más allá de la relevancia académica de un análisis de dichas posiciones, el interés y la metodología de Stumpf, como hemos visto, se centra en la descripción de la experiencia, en particular en torno al problema de la representación del espacio.

La obra se estructura a partir de dos preguntas fundamentales, a partir de las cuales se estructura metodológicamente la investigación. Una es de carácter genético y la otra de tipo conceptual: (1) ¿De donde procede una representación (Vorstellung)? (2) ¿Cómo se puede abordar científicamente una representación una vez que la tenemos? Para Stumpf ambas preguntas están íntimamente relacionadas, en tanto "la pregunta por el origen de una representación nos conduce a las partes individuales (einzelne Teile) a partir de las cuales se compone" (Stumpf 1873 , p. v). En tal sentido, no hay que perder de vista esta doble dirección (genético-conceptual) de la investigación propuesta por Stumpf. ${ }^{19}$

\footnotetext{
${ }^{19}$ Es casi inevitable pensar en un paralelo con la fenomenología de Husserl y su diferenciación entre las investigaciones 'estáticas' (conceptuales) y las 'genéticas'. De cualquier modo, este tema excede la temática del presente artículo.
} 
(1) Por un lado, la pregunta genética indaga el modo a partir del cual llegamos a tener una representación del espacio. En directa consonancia con su psicología descriptiva, Stumpf entiende el espacio que se nos presenta en nuestra experiencia, conjuntamente con diferentes cualidades, como por ejemplo la representación de color; es decir, la representación (o 'presentación') del espacio no es algo absoluto o impuesto desde la subjetividad, sino algo que viene dado a partir de la experiencia misma, en el sentido más básico y cotidiano del término. Stumpf rechaza así la idea de que el espacio es un sistema apriorístico que se impone al mundo; todo lo contrario, hay que entenderlo a partir de aquello que se nos muestra "a cada uno a partir de la vida diaria (Jedem aus dem täglichen Leben)" (Stumpf 1873, p. 2). Todas las categorías espaciales (distancia, lugar, tamaño, etc.) sólo tienen sentido a partir de la relación entre el mostrarse del mundo y de los objetos, y mi experiencia subjetiva de los mismos, que es el 'punto cero' desde donde, por ejemplo, la distancia tiene sentido. Es por esto que su investigación está íntimamente relacionada con el análisis de nuestros sentidos (la vista y el tacto fundamentalmente). En síntesis, el espacio no es algo dado con anterioridad a la experiencia, i.e. a priori, sino que 'se constituye' genéticamente a partir de ésta.

(2) Pero es la segunda pregunta formulada arriba la que nos interesa, es decir, la indagación de tipo conceptual-estructural: ¿de qué hablamos cuando hablamos de 'representación'? ¿Cómo se relaciona este concepto con otros tales como los de percepción, sensación, etc.? Es aquí, es decir, en el marco de descripción conceptual de las estructuras mismas de lo real, donde surgen y se muestran las inquietudes y los planteamientos de tipo mereológico. Siguiendo la línea de otros empiristas (tales como Locke o Hume), Stumpf presenta una taxonomía que describe el complejo mundo conceptual en torno a las categorías claves que estructuran el mundo de nuestra experiencia. A partir de esto, Stumpf desarrolla una explicación mereológica para mostrar la articulación entre conceptos tales como los de sensación (Empfindung), representación (Vorstellung), percepción (Wahrnehmung) y otros (cf. Stumpf 1873, p. 2). En un claro gesto 'brentaniano', el concepto clave en el cual se basan los demás es el de 'representación'. 


\subsection{El análisis estructural-conceptual de las 'representaciones' (Vorstellungen)}

El análisis conceptual nos presenta una amplia gama de perspectivas sobre un mismo objeto. En concreto, en el marco de las representaciones podemos distinguir lo siguiente:

(a) Una representación puede ser real, o bien de fantasía o de la memoria. La primera (la real) es para Stumpf la sensación (Empfindung) que es la más original (das Ursprünglichste): cuando oímos un sonido o vemos un color tenemos una dación directa de algo a nuestros sentidos. Las representaciones de fantasía (Phantasievorstellung) o de la memoria (Gedächtnisvorstellung) son representaciones reproductivas. ${ }^{20}$ En los dos últimos casos tenemos conciencia de algo (de un sonido o de un color, por ejemplo), pero sin dación 'en persona': es decir, no hay algo dado directamente en la experiencia, sino que, por ejemplo, nos imaginamos un sonido (o una melodía), o bien recordamos sonidos o melodías escuchadas en el pasado, i.e. en sensaciones pasadas (cf. Stumpf 1873, p. 3).

(b) Una representación puede ser concreta o abstracta. Todas las representaciones anteriores (de sensación, fantasía o memoria) son concretas, en tanto no necesitan de otra cosa para ser (volveremos sobre este punto; cf. infra, Cap. 4.1). Representaciones abstractas son por ejemplo los conceptos, en tanto un concepto no es algo independiente (como sí puede serlo un contenido sensible o de fantasía), sino que es el resultado de un proceso de abstracción a partir de algo concreto. ${ }^{21}$

(c) Las representaciones pueden ser simples o compuestas. Cualquiera de las tres clases de representaciones mencionadas arriba en (a) puede ser simple,

\footnotetext{
20 Stumpf distingue la representación 'real' (wirklich) de aquéllas que son una 'reproducción de una representación asociada' (Reproduktion einer associirten Vorstellung), i.e. las representaciones de fantasía y de la memoria. Éstas no se dan por efecto externo (äussere Einwirkung), como es en el caso de la sensación, sino que son el resultado de reproducciones asociativas de sensaciones pasadas (cf. Stumpf 1873, p. 4).

21 “Todos los conceptos son (...) abstraídos de representaciones singulares" (Stumpf 1886/87a, p. 300). En claro rechazo al realismo lógico o platónico, para Stumpf no puede haber representaciones de objetos abstractos, sino sólo de objetos concretos a partir de los cuales podemos, en un proceso intelectivo, captar, por ejemplo, conceptos en tanto partes dependientes de un todo. Volveremos luego sobre este punto (cf. infra, Cap. 4.2).
} 
como cuando escuchamos o recordamos un solo sonido, o compuesta, si se da en combinación con otros sonidos. Ahora, si observamos detenidamente nuestra experiencia real y cotidiana, aclara Stumpf que, en sentido absoluto, la mayoría de las veces nos encontramos con representaciones compuestas (y normalmente compuestas por elementos dados por los distintos sentidos) (cf. Stumpf 1873, pp. 2-3).

De este modo, podemos observar que el análisis conceptual consiste en descomponer las representaciones en sus elementos básicos, es decir, en separar ya sea las distintas representaciones que conforman un todo complejo (de representaciones), o bien los distintos componentes (partes) de una representación (todo) ${ }^{22}$; en tal sentido, Stumpf habla de una analogía con el análisis químico (cf. Stumpf 1873, p. 5; cf. supra, Cap. 2.3). En base a este método de análisis, Stumpf explica la representación del espacio (i.e. el tema central de RV): el espacio es una representación compuesta (cf. Stumpf 1873, pp. 6 y ss.) que viene siempre acompañado por cualidades (Qualitäten), como ser los datos dados por la percepción visual (los colores), o por la percepción táctil, etc. Pero, aclara Stumpf, su principal interés no recae en la descripción genética del origen del espacio, sino en el análisis estructural-conceptual de las relaciones entre espacio y cualidad(es) en la representación (cf. Stumpf 1873, p. 107). Es a partir de esta temática en torno a los diferentes modos de composición y de las relaciones entre representaciones y las partes de representaciones que Stumpf llega a su rudimentaria teoría 'mereológica', es decir, a su teoría de todos y partes, que es el punto que nos interesa aquí.

\section{La teoría de todos y partes}

\subsection{La teoría de las partes psicológicas}

La primera distinción que hay que tener en cuenta es aquélla entre partes físicas (physische Theile) y partes psicológicas (psychologische Theile) (cf.

\footnotetext{
${ }^{22}$ En el segundo tomo de TP se analiza el modo de captar las partes tonales de un todo, sea ya a partir de un mero análisis básico de captación de las partes de un todo (por ejemplo, al oír y aprehender los tonos de una melodía), sea ya a partir de un 'oír delimitante' (heraushören), que aclara y profundiza aquello dado en el análisis (cf. Stumpf 1890, pp. 6-7).
} 
Stumpf 1873 , p. 9). ${ }^{23}$ Son las segundas las que le interesan en particular a Stumpf para su análisis de la representación del espacio (los $\$ \int 5$ y 6 de RV están dedicados exclusivamente a este tema).

$\mathrm{El}$ análisis psicológico de las representaciones nos presenta diferentes posibilidades en cuanto a la conexión (o relación) entre representaciones: dependiendo de su co-pertenencia (Zusammengebörigkeit) o de su parentesco (Verwandschaft), podemos encontrar relaciones entre representaciones separables o bien entre representaciones que necesariamente dependen de otras. Las conexiones pueden ser las siguientes:

1. 'Representaciones conjuntas de contenidos contrapuestos': son aquéllas cuyos contenidos se contraponen y así se excluyen mutuamente. Stumpf da como ejemplo el caso prototípico de las 'representaciones sin objeto', como cuando hablamos de 'hierro de madera'. Sólo en la medida en que tenemos las representaciones tanto de 'madera' como de 'hierro', y que entendemos así previamente su contraposición, podemos decir a través de un juicio que 'un hierro de madera es imposible'.

2. 'Representaciones conjuntas de cualidades de diversos sentidos': son aquéllas que provienen de diversos sentidos, como las representaciones de colores y de tonos dadas al mismo tiempo. El ejemplo que da Stumpf es el de la ópera, en donde vemos imágenes y a la vez oímos sonidos.

3. 'Representaciones conjuntas de cualidades del mismo sentido': el parentesco se da aquí entre contenidos de un mismo género, por ejemplo, cuando oímos los diferentes tonos de un acorde.

4. 'Representaciones conjuntas de intensidad, cualidad, duración etc. de una misma sensación': en este caso, Stumpf subraya que la co-pertenencia es mucho más estrecha que en los casos precedentes, en tanto aquí no se trata de la conjunción de una variedad de representaciones, sino más bien de diferencias que encontramos en una misma representación (cf. Stumpf 1873, pp. 107-108).

El parentesco y la co-pertenencia de la espacialidad y la cualidad (como el color), tema central de RV, se inscribe en el marco de este último punto (4): es decir, aquí lo contenidos se encuentran más íntimamente

\footnotetext{
${ }^{23}$ En general, en RV Stumpf se refiere a lo 'psicológico' como el ámbito propio de las representaciones mentales, mientras que lo 'físico' suele hacer alusión a los aspectos 'externos', i.e. estímulos exteriores, procesos neuronales, etc. (cf. Stumpf 1873 , pp. 8, 143-144 y ss.).
} 
entrelazados que en los casos precedentes (1-3). Es entonces en el marco de la pregunta conceptual-estructural (y, reiteramos, no genética) por la relación entre las representaciones del espacio y sus componentes, que Stumpf se ve obligado a formular una serie de distinciones de índole mereológica, en aras de llegar "más profundamente a la esencia de la cosa" (Stumpf 1873, p. 108).

En función de lo anterior, podemos decir que los modos de representación conjunta de los contenidos se dividen a su vez en dos clases fundamentales en función de su 'separabilidad' (cf. Stumpf 1873, p. 109): ${ }^{24}$ (a) los contenidos independientes (selbstständige Inhalte) son "aquéllos en donde los elementos de un complejo de representaciones se pueden representar separadamente conforme a su naturaleza"; (b) los contenidos parciales (Theilinhalte $)^{25}$ son definidos por Stumpf ex negativo: son aquellos contenidos en donde no se da lo afirmado en (a). En otras palabras, a diferencia de (a), los contenidos de (b) no se pueden pensar separadamente, es decir, son contenidos que no pueden ser representados sin otros contenidos. Así, sostiene Stumpf: "no se puede pensar una cualidad de color sin una intensidad, un movimiento sin una velocidad" (Stumpf 1873, p. 109; cf. p. 113). ${ }^{26}$

Los tres primeros puntos mencionados arriba (1-3) son contenidos independientes (a), puesto que cada representación puede perfectamente pensarse sin su conexión con las otras representaciones, mientras que en el último punto (4) se trata de representaciones parciales (b), en tanto dichos contenidos no se pueden pensar separadamente, i.e. aquí la conexión es necesaria, mientras que en las primeras no lo es.

Es importante subrayar el modo en que Stumpf interpreta esta 'necesidad'. Se trata de una necesidad conforme a la 'naturaleza misma de la cosa'. Además, es una necesidad que impone limitaciones: no se pueden separar de ningún modo los contenidos parciales (ni a través de la imaginación, ni de experimentos). Stumpf es categórico al respecto: si se pudiesen representar contenidos separadamente, entonces estaríamos en presencia

\footnotetext{
${ }^{24}$ Esta distinción será luego retomada por Husserl. La referencia a Stumpf en este punto es reconocida explícitamente en el comienzo de la tercera investigación lógica (cf. Husserl 1901/1984, p. 227).

25 Es difícil dar una traducción satisfactoria del término alemán 'Theilinhalt, considerando incluso que el mismo concepto es algo ambiguo.

${ }^{26}$ Con relación a los tonos, cf. Stumpf 1890, p. 2.
} 
de contenidos independientes (a) y no de contenidos parciales (b); ergo, los contenidos parciales sólo se pueden pensar en su conjunción necesaria con otros contenidos (cf. Stumpf, pp. 109-110, 114). ${ }^{27}$ Así, en el marco de su teoría psicológica del espacio, llega Stumpf a la conclusión de que las cualidades son igualmente originarias que la representación misma del espacio, i.e. ambas no pueden pensarse separadamente (cf. Stumpf 1873, pp. 114-115, 128-129). Ahora, aclara, no se trata de una necesidad fáctica, sino de una necesidad lógica (Stumpf 1873, p. 115).

Pero lo llamativo del caso es que a pesar de tratarse de una necesidad lógica llegamos a ésta a través de la experiencia. Es la experiencia la que nos enseña a distinguir cada vez mejor las partes de un todo y a captar dichas conexiones necesarias. Así, la capacidad para captar dichas relaciones se sostiene básicamente en la experiencia concreta que tenga cada uno en una determinada área. Para entender esto, Stumpf presenta ejemplos concretos: una persona versada en música está más en condiciones que otros a la hora de distinguir entre las partes que componen un todo complejo; así, al sonar una orquesta, puede dirigir su atención a los distintos instrumentos, a los matices, etc.; otros ejemplos dados por Stumpf son los de un buen matemático o un médico experimentado (cf. Stumpf 1873, pp. 130-131). En muchos de estos casos, intervienen además el recuerdo (i.e. la memoria de las experiencias pasadas) y la fantasía (a modo de variación de imaginación, que a su vez se sustenta en la versatilidad generada por las experiencias pasadas), que nos permiten visualizar concretamente el todo y ver allí las partes que lo componen, así como la relación entre éstas y su relación con el todo. Por supuesto, como señalamos arriba, hay un límite: cuando hablamos de la separación de las partes, nos referimos a partes independientes, i.e. que pueden ser pensadas con independencia del todo. ¿Qué sucede con la separación de los contenidos parciales? ¿Cómo es posible distinguirlos?

\footnotetext{
${ }^{27}$ En este contexto, Stumpf rechaza la idea de 'asociación' de J. S. Mill, según la cual hay partes que se asocian en la experiencia y que, tras reiterarse, se vuelven 'fijas', pero que pueden ser separadas. Para Stumpf, las conexiones necesarias no son asociaciones, puesto que éstas pueden descomponerse en sus elementos, mientras que aquéllas no (cf. Stumpf 1873, pp. 110-112).
} 


\subsection{Posibilidades y limites de la captación de las partes o contenidos parciales}

En el caso, por ejemplo, de la separación de cualidades (Trennung von Qualitäten) de una sola impresión, i.e. de contenidos parciales, no puede haber ninguna percepción real de éstos (Stumpf utiliza sendos términos: 'Perception' y 'Wahrnebmung'); en estos casos, sólo la fantasía nos permite, en alguna medida, 'introducir con el pensamiento' (bineindenken) las cualidades (partes) en la sensación (todo) (cf. Stumpf 1873, pp. 131-132, 135). ${ }^{28}$ Si bien en RV no se encuentra un método lo suficientemente preciso en cuanto al modo de captación de dichas cualidades, la idea de fondo se puede dividir en dos cuestiones centrales. Primero, aquello que realmente (en sentido estricto) podemos percibir son los contenidos unitarios, independientes (cf. Stumpf 1873, p. 136). Segundo, las cualidades en tanto tales no se pueden percibir sino sólo indirectamente a partir de los modos de modificación (Veränderungsweisen) que se presentan en un contenido unitario (cf. Stumpf 1873, pp. 135-136). En otras palabras: sólo a partir de las modificaciones y variaciones (de cualidad, cantidad, etc.) podemos determinar y atribuir un determinado contenido parcial a un todo. Considerando que es metodológicamente imposible percibir un contenido parcial en sí, el modo de captación de contenidos parciales implica entonces dos momentos: por un lado, una modificación de las cualidades del contenido, y por otro lado 'introducir con el pensamiento' (bineindenken) dicha parte en el todo, i.e. determinar con el pensamiento que se trata de un cambio de cualidades que dependen de un todo y que por ello no son independientes.

Si bien este último punto es algo complejo, es fundamental, en tanto Stumpf pretende extirpar algo que considera un error común de la filosofía y de la psicología, que consiste en entender las cualidades en tanto entidades propias (eigene Entitäten), que llevan a las ilusiones psicológicas que afirman a las cualidades como contenidos en sí. En otras palabras: el error consiste en caer en la tentación de hipostasiar aquello que sólo puede pensarse en

${ }^{28}$ Es difícil encontrar una traducción del todo satisfactoria para 'bineindenken' que respete el sentido cuasi-técnico que Stumpf quiere darle a este término. En general, son numerosos los verbos que se encuentran en estas páginas de RV con el prefijo 'binein' (bineindenken, hineintragen, hineinverlegen, bineinhören), siempre con la idea de algo, i.e. las cualidades en tanto contenidos parciales, que hay que 'introducir' (o 'pensar en', o ‘transferir') en el ‘todo’ de una sensación. 
referencia a un todo. Ahora, por otra parte, esta idea de determinar o introducir con el pensamiento una cualidad en el contenido unitario, tampoco debe entenderse como una intromisión arbitraria en la cosa (i.e. no se trata en absoluto de un subjetivismo que impone condiciones formales a la cosa). Por el contrario, son los contenidos parciales mismos los que a través de sus modificaciones nos indican qué es lo que, retrospectivamente, podemos atribuir al contenido total. Como sostiene el mismo Stumpf: se trata de una "distinctio cum fundamento in re". Así, llega a la conclusión que las cualidades, conforme a su misma naturaleza, "cambian de un modo independiente, pero no existen de un modo independiente" (Stumpf 1873, p. 139). En síntesis, son dos las conclusiones: primero, se trata siempre de un único contenido con diferentes cualidades; segundo, las relaciones que atribuimos a estos contenidos no son casuales ni arbitrarias, sino que es el contenido mismo el que cambia de un modo real, y a partir de dichas modificaciones podemos captar (de un modo atributivo) las diferentes cualidades (cf. Stumpf 1873, p. 140). Traducido en clave fenomenológica: es siempre 'la cosa misma' la que se (nos) muestra y la que (nos) muestra sus modificaciones, aún cuando, en virtud del método, debamos reconocer y atribuir luego las determinaciones.

En los apuntes de clases de Stumpf de 1886/87 mencionados arriba, Stumpf parece darle un carácter metódico más preciso a las cuestiones aquí señaladas, al utilizar los conceptos de 'abstracto' y 'concreto'. Por un lado, sigue sosteniendo su idea de que sólo puede haber representaciones (Vorstellungen) de partes concretas, pero no de partes abstractas o dependientes. Pero, por otro lado, admite la posibilidad de captar o darse cuenta de las partes inseparables o abstractas: "Llamo abstracta, a aquellas partes de un contenido representacional, que pueden ser captadas, pero no representadas separadamente" (Stumpf 1886/87a, p. 300). ${ }^{29}$ Si bien es innegable que los conceptos de ‘concreción' y 'abstracción' tienen una larga historia filosófica, no obstante, su introducción en el marco de una teoría 'proto-mereológica' no deja de ser significativa, en la medida en que adquieren un significado técnico.

En los apuntes de las lecciones mencionadas (1886/87), Stumpf presenta asimismo una interesante caracterización taxonómica de las repre-

\footnotetext{
${ }^{29}$ Recordemos lo dicho arriba: los conceptos son para Stumpf abstracciones de las representaciones singulares (cf. supra, Cap. 3.2).
} 
sentaciones (Vorstellungen) en términos 'mereológicos'. En el marco del análisis lógico de los contenidos (Inhalten) de las representaciones, podemos distinguir: a) partes colectivas, que son los miembros de un todo que sumados componen un conjunto; b) partes físicas, que son aquéllas que lindan entre sí, como por ejemplo los segmentos de espacio o de tiempo; c) partes metafísicas, que son los momentos o propiedades, como v. gr. la intensidad y cualidad de un tono; d) partes lógicas, que son los géneros y diferencias, como ser 'color' y 'rojo'. Mientras que las dos primeras son 'concreta', es decir partes independientes, las dos últimas son 'abstracta', i.e. partes dependientes o, en la terminología de RV, 'contenidos parciales', en tanto no tienen existencia propia, independiente, ni podemos tener una representación directa de las mismas. Estas últimas están íntimamente entrelazadas entre sí y sólo se pueden distinguir una de otras por medio de un enfoque peculiar de la conciencia llamado 'abstracción' (cf. Stumpf 1886/87b, pp. 313-314).

\section{Desarrollo posterior de la teoría de todos y partes}

En escritos posteriores a la publicación de la tercera investigación lógica de Husserl, en particular en su Zur Einleitung der Wissenschaften (Introducción a las ciencias) de 1907, Stumpf presenta ya una teoría de todos y partes más explícita y con mayor precisión conceptual. Esta obra se presenta como una auténtica filosofía de las ciencias, que pretender explicitar la "arquitectónica del edificio de las ciencias" (Stumpf 1907, p. 3). En este marco, Stumpf ofrece una clasificación de las ciencias, así como una serie de caracterizaciones de sus diferentes métodos, conforme a la naturaleza de sus respectivos objetos de estudio. En relación con la temática que nos interesa aquí, encontramos, por un lado, una diferenciación nuclear entre las ciencias naturales y las sociales, y, por otro lado, de éstas con las 'ciencias neutrales' (neutrale Wissenschaften). Según esta caracterización, hay cuatro ciencias neutrales: tres de ellas son 'pre-ciencias' (Vorwissenschaften) y la cuarta es una 'post-ciencia' (Nachwissenschaft). El 'arbol del saber' se constituye así por tres pre-ciencias (la fenomenología, la eidología y la doctrina general de las relaciones), el núcleo central constituido por las ciencias positivas (naturales y sociales), y la post-ciencia que sería la metafísica, encargada de explicitar las leyes comunes, así como las conexiones entre los diferentes grupos de ciencias (cf. Stumpf 1907, p. 42). 
Como dijimos, en el marco de las pre-ciencias nos encontramos con tres disciplinas 'neutrales'. a) La fenomenología es la ciencia de las 'manifestaciones' (Erscheinungen), es decir, no de las funciones psíquicas, ni de los objetos correlativos, sino de las leyes mismas del aparecer; esta ciencia es la que complementa y, fundamentalmente, les da el punto de referencia (Anhaltspunkt) a las demás ciencias en tanto todas presuponen el aparecer de algo (cf. Stumpf 1907, pp. 26, 29-32; cf. Stumpf 1910, p. 186). b) La eidología es la encargada de investigar las estructuras o formaciones (Gebilde) lógicas en sentido amplio, i.e. los conceptos y su relación con las manifestaciones, que sirven de base a todas las ciencias; en otras palabras, se trata de una suerte de lógica pura (reine Logik) en el sentido en que la entendía Husserl (cf. Stumpf 1907, pp. 32-36). c) La doctrina general de las relaciones (allgemeine Verhältnislehre) es ya una formulación explícita de la teoría de todos y partes. Esta ciencia no es ni psíquica ni física. Su objeto son los conceptos relacionales que "nos son dados en el mismo sentido que [nos son dadas] las manifestaciones (Erscheinungen), con y a través de ellas (...). [N]osotros [sólo] constatamos [las relaciones], pero no las creamos" (Stumpf 1907, p. 37)..$^{30}$ En otras palabras: estas relaciones son co-captadas ('miterfassen' es el verbo utilizado) junto con las manifestaciones mismas, objetos de la fenomenología. Las relaciones pueden ser de similitud, aumento, fusiones, relaciones parciales, relaciones de dependencia lógica entre los contenidos o estadosde-cosas, etc. (cf. Stumpf 1907, p. 38).

\section{Reflexiones finales: el valor de la experiencia y de las investiga- ciones mereológicas}

Es innegable que muchas de las reflexiones mereológicas presentadas aquí encuentran claros antecedentes a lo largo de la historia de la filosofía, que van desde el mundo moderno (en particular en Locke y Berkeley), pasando por las sutiles investigaciones de los escolásticos, hasta incluso los minuciosos análisis de Aristóteles, por ejemplo, en torno al tema de las categorías. Es cierto, además, que todas estas distinciones que hemos analizado, son presentadas circunstancialmente en el marco de un contexto más amplio, que es el de los estudios psicológico-descriptivos de los 'estados

\footnotetext{
30 "Su objeto lo constituyen las relaciones más básicas (die einfachsten Verbältnisse) en todos los ámbitos fundamentales del conocer humano" (Stumpf 1907, p. 38).
} 
psicológicos', o, en términos de Brentano, de los 'fenómenos psíquicos', i.e. de las representaciones, los juicios y los estados de ánimo, por lo que, stricto sensu, no se podría hablar de una teoría explícita y sistemática de todos y partes, al menos en los primeros escritos de Stumpf. Pero, no obstante, más allá de estos puntos mencionados, nos encontramos de hecho con una caracterización conceptual del modo de presentación de contenidos o partes independientes (concretos) y de contenidos o partes dependientes o parciales (abstractos). Es decir, en la primera obra de Stumpf nos encontramos con una formulación conciente de que la realidad presenta distinciones que se deben pensar en términos de todos y partes, y que estas últimas, a su vez, se subdividen en aquéllas que pueden 'separarse' de un todo y en aquéllas que no pueden, en virtud de su dependencia de dicho todo. Son precisamente estas investigaciones las que nos permiten hablar de una mereología in statu nascendi; como hemos visto, no se trata de reflexiones aisladas y laterales, sino de reflexiones con conciencia de que el enfoque no se dirige ya a la cosa, sino a las relaciones que se presentan a partir de la cosa.

Buscar antecedentes históricos de dichas reflexiones tiene un valor meramente histórico, pero no filosófico-conceptual directo (sería más o menos como pretender restar méritos a la teoría intencional de Brentano, alegando antecedentes históricos al concepto de 'intencional' en el medioevo). Lo concreto fue que las reflexiones elementales de Stumpf en torno a todos y partes tuvo una influencia directa en el nacimiento de la primera teoría (explícita) de todos y partes en la tercera investigación lógica de Husserl, como éste mismo reconoce. ${ }^{31}$

Más allá de que a partir de sus investigaciones en torno al espacio o a los sonidos (y así también al tiempo) se haya gestado luego una 'ciencia neutral' de los todos y las partes (llamada luego 'mereología'), no debemos

\footnotetext{
${ }^{31}$ Si bien Husserl remite directamente a la obra de su director, Carl Stumpf, no se puede desconocer la eventual (y muy probable) influencia de la obra de otros grandes pensadores en su teoría de todos y partes, como por ejemplo de Bernard Bolzano y de Kazimierz Twardowski (éste último, a su vez, fue maestro de Leśniewski, el padre de la mereología). Es interesante constatar que muchos de los elementos que Husserl recibe para su teoría 'mereológica', no vienen principalmente de parte de aquellos filósofos lógicos y matemáticos (que él de hecho conocía muy bien), sino a partir de dos filósofos de la tradición de la psicología descriptiva brentaniana, como fueron Stumpf y Twardowski.
} 
olvidar el claro gesto de cuño brentaniano que estructura la base de la obra de Stumpf (y que será luego un rasgo característico de la fenomenología): es la psicología descriptiva y su análisis de la experiencia la que nos muestra a partir de 'las cosas mismas' cuáles son las estructuras fundamentales de lo real, como por ejemplo la distinción en todos y partes. En su escrito sobre las ciencias de 1907, Stumpf reconoce abiertamente este gesto 'fenomenológico' de su filosofía, al señalar que no es correcto "tomar como punto de partida, en primer lugar, el método. Los que hablan con especial predilección del método son aquellos que nunca han llevado a cabo una sola investigación basada en los hechos (sachlich). Las investigaciones radicales sobre el método se arraigan siempre en última instancia en las diferencias de los objetos" (Stumpf 1907, p. 4). Esta última oración es categórica: no debemos 'imponer' nada a la cosa, sino que, todo lo contrario, debemos dejarnos guiar por ésta y estar atentos a lo que nos muestra.

Además, Stumpf reconoce que debido al extraordinario grado de abstracción (außerordentliche Abstraktheit) no es conveniente comenzar (en un sentido de anterioridad metodológica o histórico-genética, i.e. no en sentido de anterioridad lógica) con este tipo de estudios 'mereológicos'. Todo lo contrario, es aconsejable comenzar con investigaciones focalizadas en diversos 'objetos' de estudio (el espacio, el tiempo, los sonidos, las percepciones, etc.), sirviéndose sólo de las relaciones mereológicas de un modo implícito, sin reflexionar sobre ellas; sólo luego, a partir de dichos estudios, es aconsejable hacer explícitas dichas estructuras básicas inherentes a la realidad (cf. Stumpf 1907, p. 39).

Estas pre-ciencias -y en particular la mereología que es la que nos interesa aquí- constituyen (y ahora sí en un sentido de anterioridad lógica) una suerte de 'atrio' o incluso de 'organon', en tanto todo objeto y todos los conceptos relacionales de cualquier tipo de ciencia reciben su legitimación a partir de éstas (cf. Stumpf 1907, p. 39). Así, todo concepto básico y toda relación (entre conceptos, objetos, contenidos, estados-de-cosas, etc.) presuponen lógicamente lo dado por estas 'ciencias neutrales', en particular, por la 'teoría general de las relaciones'. La relevancia de esta última es invaluable: nos permite evitar errores categoriales, hipóstasis infundadas, generalizaciones equívocas u otros falsos procedimientos metodológicos (falaces o, sencillamente, erróneos), entender el alcance y significación de conceptos fundamentales tales como los de similitud, fusión, etc. En síntesis, es en 
principio infinito el campo de aplicación de dicha ciencia mereológica, en tanto toda ciencia depende (i.e. está condicionada lógicamente) de ésta.

Por supuesto, es menester enfatizar una vez más la idea de anterioridad 'lógica' a diferencia de la anterioridad 'fáctica'. Como dijimos, Stumpf desaconseja comenzar las investigaciones con estas ciencias, debido a su complejidad, por lo que su anterioridad lógica no siempre coincide con su anterioridad fáctica. El camino emprendido por Stumpf es por ello muy similar al de la fenomenología de Husserl ${ }^{32}$ : siempre hay que volver a las cosas mismas, a nuestra experiencia cotidiana, diaria, en el sentido más elemental del término, a los ejemplos del día a día, a la mirada atenta de aquellos detalles que se nos muestran en el aparecer mismo de las cosas y que quizás no todos pueden ver. Sólo a partir de allí lograremos captar las estructuras invariantes de la realidad, de su fenomenalidad, así como las estructuras formales básicas de todos y partes. Esta última reflexión bien podría haber sido firmada por Edmund Husserl o por el mismo Carl Stumpf.

\footnotetext{
${ }^{32}$ En un sentido análogo al de Husserl, sostiene Stumpf en una conferencia que la filosofía "osa acercarse a lo más difícil, a lo más sublime, y a lo último, sólo en progresos infinitos a través del tratamiento de los conceptos de la experiencia" (Stumpf 1910, p. 192).
} 


\section{Bibliografía}

Husserl, Edmund (1901/1984), Logische Untersuchungen. Zweiter Band. I. Teil. Husserliana XIX/1. The Hague: Nijhoff.

Rollinger, Robin (1999), Husserl's Position in the School of Brentano. Dordrecht: Kluwer.

Rollinger, Robin (2008), Austrian Phenomenology. Frankfurt: Ontos Verlag. Schuhmann, Karl (1996), "Carl Stumpf”, en Albertazzi, L. / Libardi, M. / Poli, R. (eds.), The School of Franz Brentano. Dordrecht: Kluwer; pp. 109-129. Smith, Barry (1994), Austrian Philosophy. The Legacy of Franz Brentano. Chicago: Open Court.

Smith, Barry (ed.) (1982), Parts and Moments: Studies in Logic and Formal Ontology. München / Wien: Philosophia Verlag.

Stumpf, Carl (1873), Über den psychologischen Ursprung der Raumvorstellung. Leipzig: Verlag von S. Hirzel.

Stumpf, Carl (1883): Tonpyschologie. Band I. Leipzig: Verlag von S. Hirzel.

Stumpf, Carl (1886/87a), Syllabus for Psychology. Trad. R. Rollinger, en Rollinger 1999.

Stumpf, Carl (1886/87b), Syllabus for Logic. Trad. R. Rollinger, en Rollinger 1999.

Stumpf, Carl (1890), Tonpyschologie. Band II. Leipzig: Verlag von S. Hirzel.

Stumpf, Carl (1907), Zur Einteilung der Wissenschaften. Berlin: Verlag der Königl. Akademie der Wissenschaften.

Stumpf, Carl (1910), Philosophische Reden und Vorträge. Leipzig: Verlag von Johann Ambrosius Bath.

Stumpf, Carl (1919), "Erinnerungen an Franz Brentano", en Kraus, O., Franz Brentano. Zur Kenntnis seines Lebens und seiner Lehre. München: C.H. Beck'sche Verlagsbuchhandlung; pp. 85-149.

Recibido: 08/2014. Aceptado: 11/2014. 
